\title{
A CONTEXT-AWARE APPROACH TO INFER TRUST IN PUBLIC KEY INFRASTRUCTURES
}

\author{
Fabio Di Vito, Paola Inverardi, Giovanna Melideo \\ Dipartimento di Informatica, University of L'Aquila, \\ via Vetoio, 67010 L'Aquila, Italy. \\ \{divito, inverard, melideo\}@di.univaq.it
}

\begin{abstract}
A fundamental issue for a real uptake of e-commerce regards trust among parties in authentication infrastructures based on public key certificates (PKI): a reference entity $e$, before establishing an e-commerce relation with a (previously unknown) target entity $t$, would like to know to which degree it can trust $t$.

In this paper we investigate algorithmic techniques to efficiently support a context-based approach to establish trust degrees among entities.
\end{abstract}

Keywords: public key certification, trust, recommendations, security services

\section{INTRODUCTION}

Determining in a reliable way the legitimacy of certificates and the authenticity of public keys appears to be a basic ingredient for executing transactions securely in any large-scale open system. More and more often, the realization of e-business support system is being based on the use of PKI (Public Key Infrastructure) for the emission of digital certificates guaranteeing the identity of users in transactions $[4,7,8,9,12]$. A PKI refers to an infrastructure for distributing public keys, where the authenticity of public keys is certified by Certification Authorities ${ }^{1}$ (CAs). In order for two users to verify the authenticity of public keys of each others' it is sufficient that there exists a certification path between them, that is a sequence of certificates (correspondingly, a sequence of CAs issuing them) which together with the public key of the initial certificate in the path can be processed to obtain the public key contained in the final certificate in the path.

${ }^{1}$ Certification authorities are also called trust third parties or introducers.

The original version of this chapter was revised: The copyright line was incorrect. This has been corrected. The Erratum to this chapter is available at DOI: 10.1007/978-0-387-35696-9_19 
In this paper we are not concerned with software aspects of a PKI, i.e., we do not consider the problem of how to propagate authenticity of public-keys by certificates. We rather analyze a fundamental issue underlying authentication problems in large e-commerce infrastructures, i.e., trust, which is a basic PKI characteristic: on the one hand, a user is required to trust the CA's certificates (how a user trusts a CA will define how much trust it has in using the CA's certificates for secure communications) ${ }^{2}$; on the other one, an entity must be trustable to protect its private key.

Trust may arise as a direct relationship between CAs and users (direct trust) or as result of a reference being provided by someone else (recommendation trust). This involves a question of transitivity of trust, i.e., "if I trust a party, do I therefore trust a further party that it recommends, endorses, or introduces me to?"

Propagating authenticity of public-keys by certificates is quite straightforward. In contrast, it is less obvious how trust should be established and propagated. In this paper we propose an approach to infer and increase trust among parties in authentication infrastructures based on public key certificates.

More precisely, the problem that we consider is the following. A reference entity $e$ (e.g., an end user or a CA) want to establish an e-commerce relation with a (previously unknown) target entity $t$. Before entering into this kind of relationship, $e$ would like to know to which degree it can trust $t$.

A PKI can be modelled as a weighted directed graph, called trust graph, where the weight of a directed edge from $x$ to $y$ expresses the degree of $x$ 's trust in $y$. In terms of the trust graph, then, this means $e$ has to establish an edge from itself to $t$ whose weight is the degree of its trust in $t$. If both $e$ and $t$ have their identities certified by the same CA then it is easy for $e$ to determine the weight of $(e, t)$. Otherwise, $e$ has to search the trust graph for paths (i.e., sequences of edges) leading to $t$. Clearly, there are two contrasting objectives: (i) the search should produce a path to $t$ providing a large enough degree of trust - otherwise the e-commerce relation cannot be established on a reliable enough base, (ii) the search process should be efficient, both in time spent and in computational resources used. In fact, searching the trust graph in an unrestricted manner can in general lead to a waste of time and computational resources, since the tendency is to have larger and larger connected trust graphs. The search process has therefore to be guided towards more promising directions of exploration.

\footnotetext{
${ }^{2}$ The degree to which users can trust the binding between the public-key and the owner identity stored in a certificate depends on several factors, including the practices followed by the $\mathrm{CA}$ in verifying the identity of the owner, the CA's operating policy, procedures and security controls, the owners obligations e.g. regarding secure storage of the private key, and legal obligations of the CA.
} 
To formalize this approach we use an approach based on context-aware computing [6]. Informally speaking, the context for an entity $e$ is a way of incrementally representing the degree of trust that $e$ can infer in farther and farther nodes as the trust graph is explored. The context is therefore, in some sense, the viewpoint of $e$ on the entire trust graph. Conceptually, it is similar to a user view of the enterprise database in the DBMS area.

More formally, the context for an entity $e$, which is trying to establish the degree of its trust in another entity $t$, is an incrementally built directed graph, rooted at $e$. When the context reaches $t$ then the weight of the (transitive) edge from $e$ to $t$ represents the degree of trust.

In this paper we focus on the problem of efficiently building the context for an entity $e$ in the simple situation where the context building process only works on the PKI graph. It is clear that, in general, context building processes may be activated by various entities in a completely independent way. Hence, when the process activated by $e$ reaches an entity $x$ it might find that $x$ has a current context and it might make use of it. But this introduces further complexity, since if the process activated by $x$ subsequently updates the context of $x$ this may affect the results computed by the process activated by $e$.

\section{THE MODEL}

\subsection{A GENERAL DESCRIPTION OF PKI}

A public key certificate is a digital document that contains the name and the public key of an entity (a server, an application program, etc.). The purpose of a certificate is to link in an unambiguous way a public key together with the owner identity. The core PKI is concerned with issuing and managing certificates and consists of Certification Authorities (CAs). A CA digitally signs with its private key the public key certificates that itself has issued.

An entity $e$ can use a certificate issued by a CA $X$ for a target entity $t$ if and only if: (i) $e$ knows $X$ 's public key, and is convinced of its authenticity; (ii) $e$ trusts $X$ to be honest and to correctly authenticate the owner of a public key before signing it. Obviously, most PKIs permit CAs to certify other CAs. Thus, if $e$ does not know $X$ 's public key, the first condition can be satisfied by using a certificate for $X$ 's public key issued by a CA $Y$. This process can be iterated, thus making use of a chain of certificates (and CAs issuing them), i.e., a certification path ${ }^{3}$. If $e$ trusts every $\mathrm{CA}$ on the path, then perhaps it can believe that a proper name-to-key binding has been obtained. However, the trust in this binding relies on the correctness of every CA in the path; if any $\mathrm{CA}$ in a path incorrectly authenticates the next $\mathrm{CA}$, then the user can be misled

\footnotetext{
${ }^{3}$ Many systems - DSSA, SPX, PEM, PGP - resort to authentication by a path, or chain, of CAs
} 
regarding the authentication of subsequent authorities in the path, including the target $t$.

\subsection{THE TRUST GRAPH}

The described scenario can be modelled as a directed graph with weighted edges $G=(V, R, T)$, called trust graph, where $V$ is the set of nodes, and $R$ and $T$ are two sets of directed edges. Nodes represent entities and edges in $R$ and $T$ represent two different kinds of trust relationships between entities. Weights are values measured on a continuous scale in $[0,1]^{4}$.

Namely:

- Each edge $(A, e) \in R$ (reliability edge, or r-edge) between a CA $A$ and an entity $e$ (based on the PKI infrastructure) means that $A$ issues certificates for $e$ (hence $e$ knows the public key of $A$ ). A weight $r_{A, e}$ associated with a directed edge $(A, e) \in R$ is called a "reliability degree" and represents the reliability of the certification process through which a certificate for $e$ 's public key was issued by $A$.

- An edge $(e, A) \in T$ (trust edge, or $t$-edge) from an entity $e$ to a CA $A$ specifies the degree $t_{e, A} \in[0,1]$ of $e$ 's trust ("trust degree") in the certificates issued by $A$.

Figure 1 shows an example of trust graph, where entities are represented a rectangular or rounded nodes (the former representing CAs), r-edges are drawn as arrows from CAs to entities and t-edges are drawn as dashed arrows from entities to CAs.

The problem to be solved can therefore be expressed in terms of this model as follows: given a reference entity $e$ in the trust graph and a threshold confidence value $\beta \in(0,1]$, find the set of the entities $\widehat{V} \subseteq V$ such that the degree of $e$ 's confidence (inferred trust) in each entity $y \in \overline{\widehat{V}}$ (i.e., the weight $c_{e, y}$ of the path from $e$ to $y$ ) is greater than $\beta$ and maximized.

\section{THE ALGORITHM}

The general idea of the algorithm is the following. Starting from the entity $e$, the trust graph is explored in phases, by virtually exploring at each phase a larger and larger neighborhood. From each exploration a set of potential candidates for the next exploration is built as follows: candidates of the current exploration are examined and their adjacents in the trust graph are retained as candidates for the next exploration only if (i) they have not yet been reached,

\footnotetext{
${ }^{4}$ For instance, in [8] these values are interpreted as probabilities in a well-defined random experiment.
} 


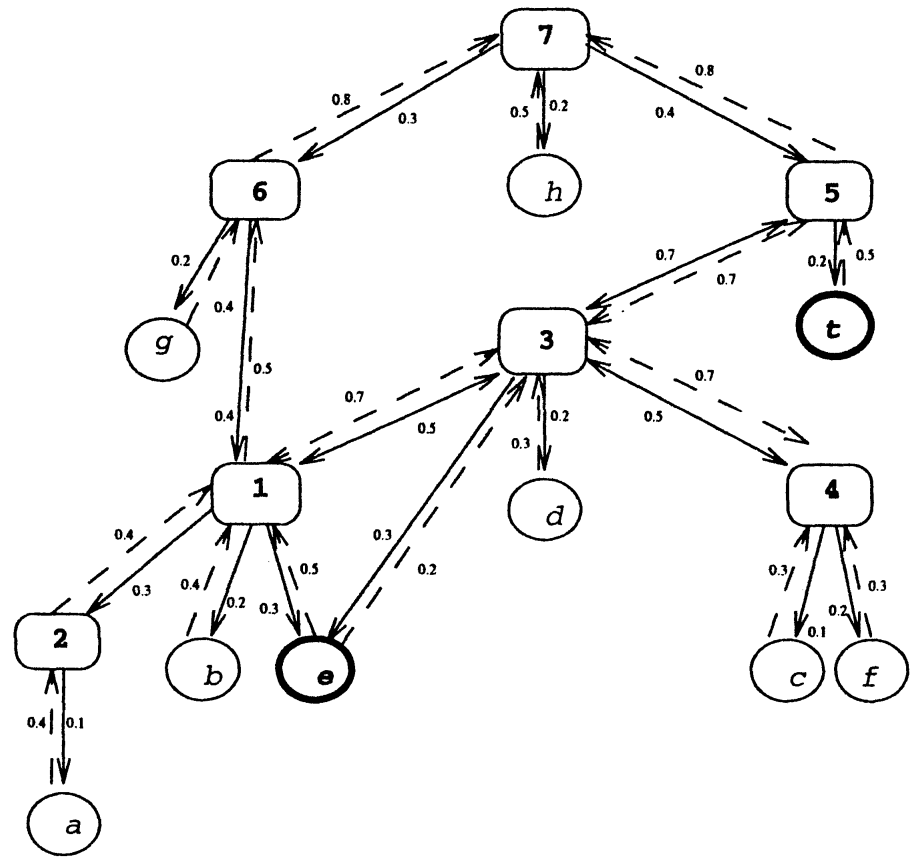

Figure 1 An example of trust graph

and (ii) the degree of $e^{\prime}$ confidence in them is above a given minimum threshold. To give efficiency to the process, candidates of the current exploration are considered in order of their value of confidence, so that more confident nodes are examined first.

Each transitive edge from $e$ to a node $y$, called c-edge, in its context is associated with a value $c_{e, y}$, called the confidence degree or $c$-degree. We denote the set of such transitive edges as $\widehat{C}$. At each phase $e$ enlarges its context by inserting new nodes as long as its confidence in them remains above a given threshold.

The computation of the edge's weight $c_{e, y}$, expressing the inferred degree of $e$ 's confidence in $y$, is executed according to the following general idea [10]: if a confidence degree $c_{e, x}$ exists between $e$ and $x$, and $x$ is a candidate of the current exploration, and during its exploration an edge $(x, y) \in T \cup R$ is found with trust degree $t_{x, y}$ and/or reliability degree $r_{x, y}$, then the value $c_{e, y}$ is computed by a suitable function of $c_{e, x}, t_{x, y}$ and $r_{x, y}$.

Let $\widehat{G}=(\widehat{V}, \widehat{R}, \widehat{T}, \widehat{C})$ be the context graph at a generic step, where $\widehat{V} \subseteq V$ is the set of nodes, and $\widehat{R} \subseteq R, \widehat{T} \subseteq T$ and $\widehat{C}$ are sets of weighted directed edges. Without loss of generality, let us suppose that the trust/reliability/confi- 
dence degree of $x$ in $y$ is zero if there is no t-edge/r-edge/c-edge $(x, y)$ in the graph. In the rest of the paper we use the following notation:

- for each node $x, N(x)$ denotes the set of neighbours of $x$ in the trust graph, i.e., $N(x)=N_{t}(x) \cup N_{r}(x)$ where $N_{t}(x)=\{Y \in V \mid(x, Y) \in$ $T\}$ and $N_{r}(x)=\{Y \in V \mid(Y, x) \in R\}$;

- $\widehat{V}_{0}$ is the set of nodes in $\widehat{V}$ whose neighbours have been visited: initially $\widehat{V}_{0}:=\emptyset$.

We call active nodes the set of nodes in $\widehat{V} \backslash \widehat{V}_{0}$ and inactive nodes the set of nodes in $\widehat{V}_{0}$.

Let $e$ be the reference entity in the trust graph, $t$ be the target entity and $\beta$ be a given threshold confidence value in $(0,1]$. The initial context of $e$ is $\widehat{G}=(\{e\}, \emptyset, \emptyset,\{e, e\})$ where $c_{e, e}=1$. In fact, it is reasonable to assume that the only node in the initial context is the (active) reference entity $e$ and to suppose that the degree of $e$ 's confidence in itself is maximum.

At each phase the most confident node $x$ among active nodes $\widehat{V} \backslash \widehat{V}_{0}$ is chosen. If the confidence degree $c_{e, x}$ is above the given threshold $\beta$ then every adjacent node of $x$ in the trust graph is inserted as active node in $\widehat{V}$ for the next phase and $x$ is inserted in the set of inactive nodes $\widehat{V}_{0}$.

Each time a node $y \in N(x)$ is inserted into the context as an active node, the weights $c_{x, y}, c_{e, y} \in[0,1]$ have to be inferred by a suitable function of $c_{e, x}, t_{x, y}$ and $r_{x, y}$. More precisely, $c_{e, y}=c_{e, x} c_{x, y}$ where $c_{x, y}$ is computed as follows:

- in the case of cross-certification between $x$ and $y$, there are both a t-edge and a r-edge from $x$ to $y$, i.e., $(x, y) \in T \cap R$, and we assign to $c_{x, y}$ the "average confidence degree", i.e.,

$$
c_{x, y}=\frac{p_{1} t_{x, y}+p_{2} r_{x, y}}{2}
$$

where $p_{1}$ and $p_{2}$ are two parameters ranging in $[0,1]$ whose values depend on the specific politics used;

- in other cases $c_{x, y}$ is actually the weight of the only edge from $x$ to $y$ in the trust graph, i.e.,

$$
c_{x, y}=t_{x, y}+r_{x, y}
$$

The execution stops when (i) the search has produced a path from $e$ to $t$, or (ii) every node into the context has been explored (i.e., $\widehat{V}=\widehat{V}_{0}$ ), or (iii) the confidence degree of remaining active nodes in $\widehat{V} \backslash \widehat{V}_{0}$ is under the given threshold $\beta$. Now we are ready to present the algorithm. 
Procedure EnlargeContext $\left(e \in V, t \in V, \beta \in(0,1], p_{1} \in[0,1], p_{2} \in[0,1]\right)$; begin

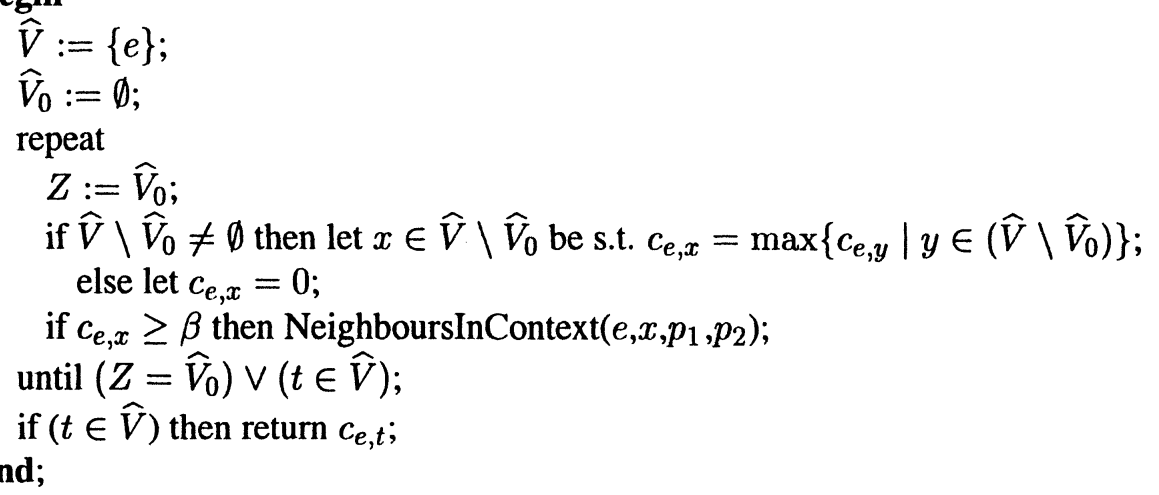

Procedure NeighboursInContext $\left(e, x \in \widehat{V}, p_{1} \in[0,1], p_{2} \in[0,1]\right)$;

begin

$$
\begin{aligned}
& \widehat{V_{0}}:=\widehat{V}_{0} \cup\{x\} \\
& \widehat{V}:=\widehat{V} \cup N(x) \\
& \widehat{T}:=\widehat{T} \cup\left\{(x, y) \mid y \in N(x) \cap N_{t}(x)\right\} ; \\
& \widehat{R}:=\widehat{R} \cup\left\{(x, y) \mid y \in N(x) \cap N_{r}(x)\right\} \\
& \widehat{C}:=\widehat{C} \cup\{(e, y) \mid y \in N(x)\} \\
& \text { for each } y \in N(x) \text { do Weight }\left(e, x, y, p_{1}, p_{2}\right) ;
\end{aligned}
$$
end;

Procedure Weight $\left(e, x, y \in \widehat{V}, p_{1} \in[0,1], p_{2} \in[0,1]\right)$;

\section{begin}

$$
\begin{aligned}
& \text { if }(x, y) \in(T \cap R) \\
& \quad \text { then } c_{x, y}:=\frac{p_{1} t_{x, y}+p_{2} r_{x, y}}{2} \\
& \text { else } c_{x, y}:=t_{x, y}+r_{x, y} \\
& \text { if } x \neq e \text { then } c_{e, y}:=\max \left(c_{e, y}, c_{e, x} \cdot c_{x, y}\right) \text {; }
\end{aligned}
$$

end;

An example. In the following the execution of three phases of the EnlargeContext procedure is described, when $p_{1}=p_{2}=1$ and the trust graph in Figure 1 is the input. The graph in Figure 5 shows the context graph obtained after three phases:

Phase 0 The initial context is $\widehat{G}=(\widehat{V}=\{e\}, \widehat{R}=\emptyset, \widehat{T}=\emptyset, \widehat{C}=\{e, e\})$ (see Figure 2);

Phase 1 Initially, as first node to be explored, only $e$ can be chosen, so its neighbours are inserted into the context as active nodes, i.e., $\widehat{G}=(\widehat{V}=$ 


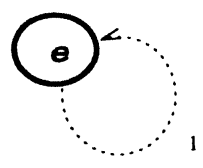

Figure 2 Initial context: $\widehat{V}=\{e\}$ and $c_{e, e}=1$

$\{e, 1,3\}, \widehat{R}=\emptyset, \widehat{T}=\{(e, 1),(e, 3)\}, \widehat{C}=\{(e, e),(e, 1),(e, 3)\})$. Then, $e$ becomes inactive, that is $\widehat{V}_{0}=\{e\}$ (see Figure 3 ).

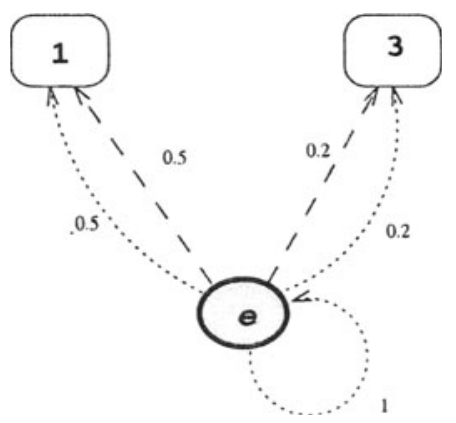

Figure 3 Phase 1: the entity $e$ has been explored

Phase 2 Node 1 is chosen in $\widehat{V} \backslash \widehat{V}_{0}=\{1,3\}$ as the more confident, and its neighbours are inserted into $\widehat{V}$. Then $\widehat{G}=(\widehat{V}, \widehat{R}, \widehat{T}, \widehat{C})$, where:

- $\widehat{V}=\{e, 1,3, b, 2,6\}$,

- $\widehat{R}=\{(1, b),(1,2),(1,6),(1,3)\}$,

- $\widehat{T}=\{(e, 1),(e, 3),(1,3)\}$,

- $\widehat{C}=\{(e, e),(e, 1),(e, 3),(1, b),(1,2),(1,6),(1,3),(e, b),(e, 2)$, $(e, 6)\}$.

Note that node 3 was already into the context and the confidence degree $c_{e, 3}$ is incremented. Also, node 1 becomes inactive, that is $\widehat{V}_{0}=\{e, 1\}$. Figure 4 shows the situation at the end of this phase.

Phase 3 Node 3 is chosen in $\widehat{V} \backslash \widehat{V}_{0}=\{3,6,2, b\}$ as the most confident, and its neighbours are inserted into $\widehat{V}$, that is $\widehat{G}=(\widehat{V}, \widehat{R}, \widehat{T}, \widehat{C})$, where:

- $\widehat{V}=\{e, 1,3, b, 2,6, d, 4,5\}$,

- $\widehat{R}=\{(1, b),(1,2),(1,6),(1,3),(3, d),(3,4),(3,5)\}$

- $\widehat{T}=\{(e, 1),(e, 3),(1,3),(3,4),(3,5)\}$,

- $\widehat{C}=\{(e, e),(e, 1),(e, 3),(1, b),(1,2),(1,6),(1,3),(e, b),(e, 2)$, $(e, 6),(3, d),(3,4),(3,5),(e, d),(e, 4),(e, 5)\})$. 


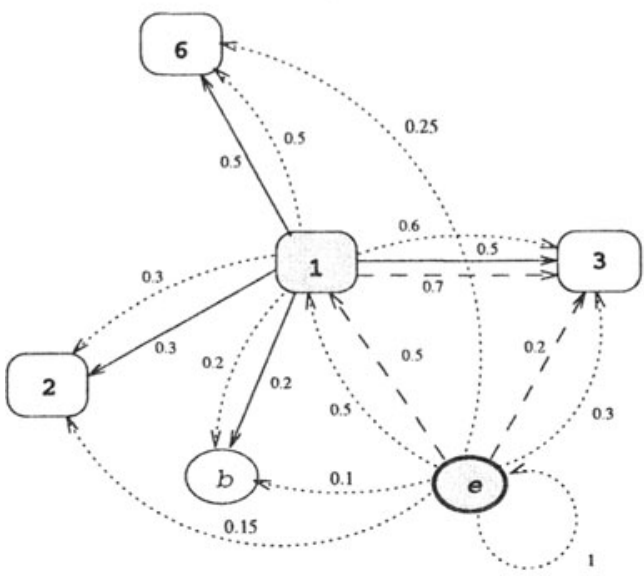

Figure 4 Phase 2: the entity 1 has been explored

Afterwards, node 3 becomes inactive, that is $\widehat{V}_{0}=\{e, 1,3\}$ and its confidence degree has reached the maximum (see Figure 5).

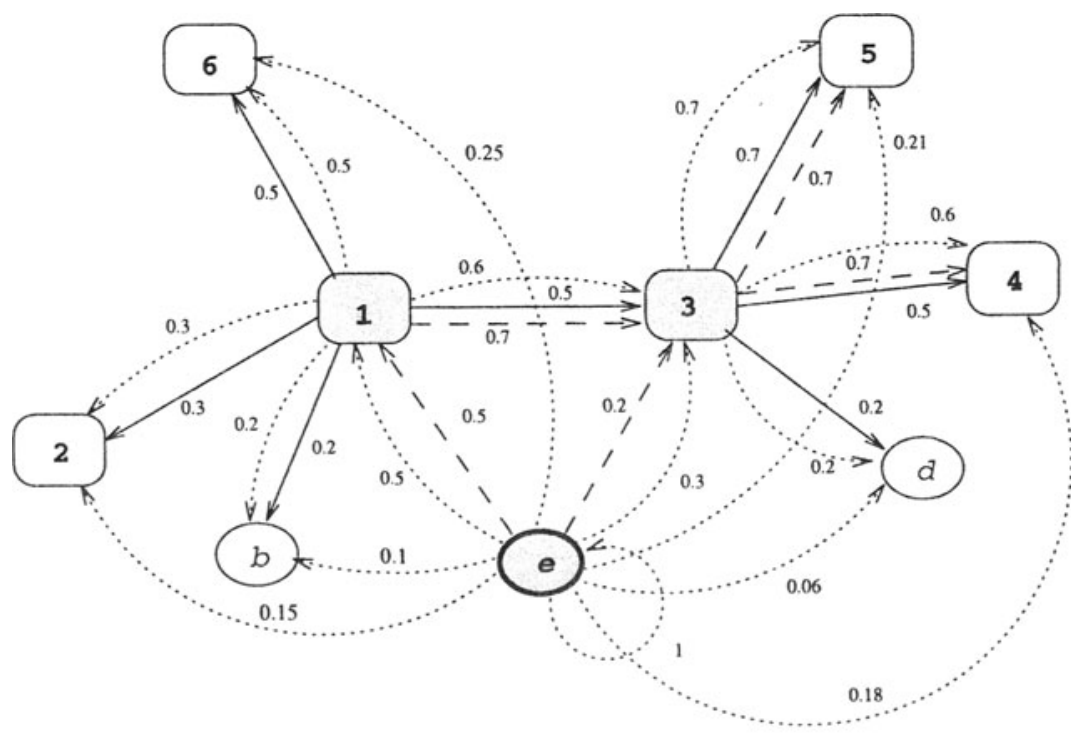

Figure 5 Phase 3: the entity 3 has been explored 


\section{IMPROVING THE ALGORITHM}

\subsection{A BASIC IMPROVEMENT}

Previous approach does not take into account the fact that the degree of $x$ 's confidence in a neighbour $y$ could not be large enough to compute a value $c_{e, y}$ of at least $\beta$. Then, a more efficient version of the algorithm is obtained by not including into the context (that is, by not considering as possible candidates for next phases) the neighbours $y$ of $x$ such that $c_{e, y}<\beta$.

Given two parameters $p_{1}, p_{2} \in[0,1]$ and an entity $y \in N(x)$, the degree $c_{x, y}$ of $x$ 's confidence in $y$ is computed as defined by Equations (1) and (2). Then, if $x$ is the active node with maximum confidence degree (with respect to the reference node $e$ ), the approach is improved by inserting into the context only the neighbours $y$ of $x$ such that $c_{x, y} \geq l(e, x, \beta)$, where $l(e, x, \beta)=\frac{\beta}{c_{e, x}}$ is called the threshold trust degree "local at $x$ ". This improvement guarantees that the degree of $e$ 's confidence in each node into the context is above the "global" threshold value $\beta$.

Let $N^{\alpha}(x)$ be denote the set of neighbours $y$ of $x$ such that $c_{x, y} \geq \alpha$. In the following we describe the improved version of the algorithm "EnlargeContext" based on previous observations.

Procedure EnlargeContext $2\left(e \in V, t \in V, \beta \in(0,1], p_{1} \in[0,1], p_{2} \in[0,1]\right)$; begin

$$
\begin{aligned}
& \widehat{V}:=\{e\} ; \\
& \widehat{V}_{0}:=\emptyset ; \\
& \text { repeat }
\end{aligned}
$$

let $x$ be the entity in $\widehat{V} \backslash \widehat{V}_{0}$ such that $c_{e, x}=\max \left\{c_{e, y} \mid y \in\left(\widehat{V} \backslash \widehat{V}_{0}\right)\right\}$;

NeighboursInContext2 $\left(e, x, \beta, p_{1}, p_{2}\right)$;

until $\left(\widehat{V}=\widehat{V}_{0}\right) \vee(t \in \widehat{V})$;

if $(t \in \widehat{V})$ then return $c_{e, t}$; end;

Procedure NeighboursInContext2(e, $\left.x \in \widehat{V}, \beta \in(0,1], p_{1} \in[0,1], p_{2} \in[0,1]\right)$; begin

$$
\begin{aligned}
& \widehat{V}_{0}:=\widehat{V}_{0} \cup\{x\} ; \\
& \alpha:=\frac{\beta}{c_{e, x}} ; \\
& \widehat{V}:=\widehat{V} \cup N^{\alpha}(x) ; \\
& \widehat{T}:=\widehat{T} \cup\left\{(x, y) \mid y \in N^{\alpha}(x) \cap N_{t}(x)\right\} ; \\
& \widehat{R}:=\widehat{R} \cup\left\{(x, y) \mid y \in N^{\alpha}(x) \cap N_{r}(x)\right\} ; \\
& \widehat{C}:=\widehat{C} \cup\left\{(e, y) \mid y \in N^{\alpha}(x)\right\} ;
\end{aligned}
$$

for each $y \in N^{\alpha}(x)$ do Weight $\left(e, x, y, p_{1}, p_{2}\right)$;

end; 
An example. Figure 6 shows the context graph obtained after two phases of the execution of the EnlargeContext 2 procedure on the trust graph in Figure 1, when the threshold trust value is $\beta=0.2$ and $p_{1}=p_{2}=1$. The context graph is $\widehat{G}=(\widehat{V}, \widehat{R}, \widehat{T}, \widehat{C})$, where: $\widehat{V}=\{e, 1,3,6\}, \widehat{R}=\{(1,6),(1,3)\}$, $\widehat{T}=\{(e, 1),(e, 3),(1,3)\}, \widehat{C}=\{(e, e),(e, 1),(e, 3),(1,6),(1,3),(e, 6)\})$. In fact, unlike the previous case depicted in Figure 4, nodes $b$ and 2 cannot be inserted into the context, because $r_{1,2}, r_{1, b}<\frac{\beta}{c_{e, 1}}=0.4$.

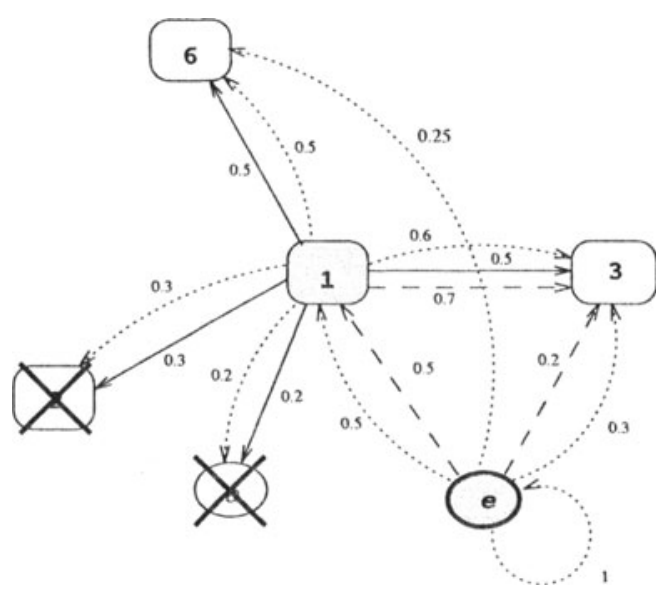

Figure 6 Phase 2 when executing the EnlargeContext2 procedure

Then, when node 3 is explored, the only adjacent node in $N(3)=\{d, 4,5\}$ which can be inserted into the context is 5 (see Figure 7). In fact, with regards nodes $d$ and 4, they cannot be inserted into the context, because $0.3, \frac{0.7+0.5}{2}<$ $\frac{\beta}{c_{e, 3}}=0 . \overline{6}$. Hence, the context graph $\widehat{G}$ becomes $\widehat{G}=(\widehat{V}, \widehat{R}, \widehat{T}, \widehat{C})$, where: $\widehat{V}=\{e, 1,3,6,5\}, \widehat{R}=\{(1,6),(1,3),(3,5)\}, \widehat{T}=\{(e, 1),(e, 3),(1,3),(3,5)\}$, $\widehat{C}=\{(e, e),(e, 1),(e, 3),(1,6),(1,3),(e, 6),(3,5),(e, 5)\}$.

\subsection{AN ITERATIVE VERSION}

Both presented versions of EnlargeContext procedure can fail and terminate without producing a confidence path from $e$ to the target entity $t$ since no path producing a confidence degree $c_{e, t} \geq \beta$ exists in the trust graph. To give efficiency to the process, we propose an iterative version of the algorithm which, in such a case, decrements the threshold trust degree $\beta$ (according to a set of rules that may be orthogonal to the ones used by the search process) and resumes the process of enlargement of the context. 


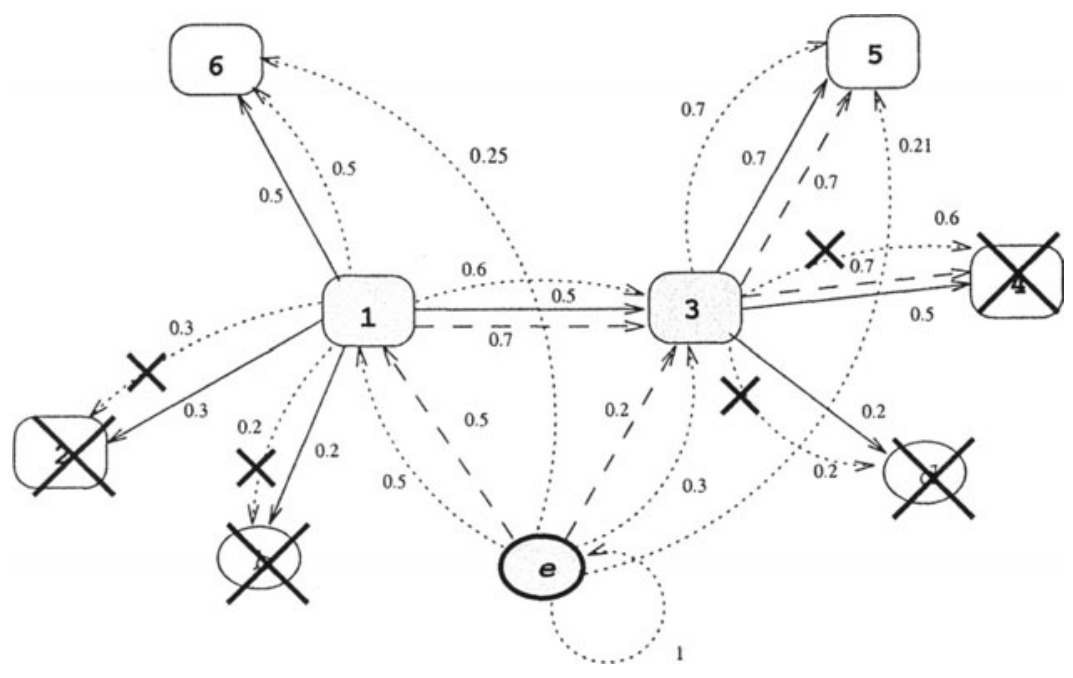

Figure 7 Phase 3 when executing the EnlargeContext2 procedure

In the following we therefore describe such an iterative algorithm which extends the "static" EnlargeContext procedure.

Procedure Iterative-EnlargeContext $\left(e, t \in V, \beta \in(0,1], p_{1}, p_{2} \in[0,1]\right)$; begin

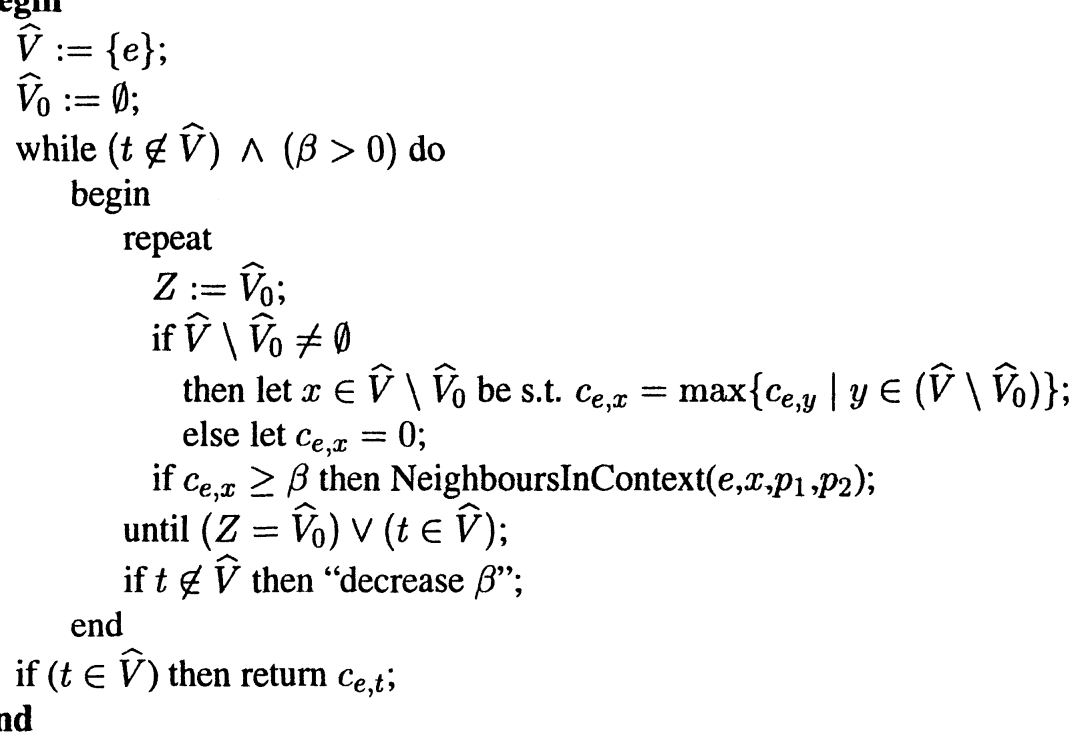

For shortness we will omit the iterative version which extends the EnlargeContext2 procedure. Let us remark that in this case it is necessary to track at 
each phase the set of inactive nodes $x$ in $\widehat{V}_{0}$ whose neighborhood has not been completely visited. Namely, a set $\widehat{Z}_{0} \subseteq \widehat{V}_{0}$ has to be composed of inactive nodes $x$ for which $N(x) \backslash N^{\alpha}(x) \neq \emptyset$ where $\alpha=\frac{\beta}{c_{e, x}}$. Each time the threshold trust value $\beta$ is decreased, the procedure has to re-explore every node in $Z_{0}$.

\section{RELATED WORK}

Many researchers have analyzed the concept of trust from different viewpoints: from sociology to risk management, from cognitive theory to economics and game theory. In the common sense meaning of the word, an entity $u$ will only establish a transaction with an entity $v$ if the level of trust $u$ has in $v$ exceeds some threshold which usually depends on both $u$ and $v$ and the context of the interaction [3].

From the point of view of economic theory, the importance of enhancing trust in public key infrastructures is supported by the interesting results in [1], where it is proved that $(i)$ a market in which agents are trusted to the degree they deserve to be trusted is as efficient as a market with complete trustworthiness and that (ii) distrust could significantly reduce market efficiency. In other words, what really counts is not the actual level of trustworthiness, but the accuracy of individual estimates of trust. Therefore [1] concludes that "the problem of learning accurate trust estimates is especially important for market efficiency".

The issue of enhancing trust is a recognizably important one also in electronic communities. In fact, in this kind of communities it is harder for people to choose which one, among the many similar providers of an e-service, is the most reliable one. In the physical world, you have commercial reputations built over years and years of reliable transactions. But in the quickly changing scenarios characterizing virtual communities reputations is a much more elusive concept.

Standard remedies to this are based on the use of trusted third parties and the whole PKI approach stems from this foundation. However, some finds this approach still weak in the case, for example, of highly dynamic communities where new users enter continually and it may difficult to agree on the use of a common trusted third party or of set of them [4]. Indeed, the research discussed in our paper aims exactly at allowing to a set of entities to enter into reliable commercial relations even if each of them has its own trusted third party. If one wants anyhow to avoid to resort to trusted third parties, then an alternative is the one suggested in [4], based on the use of cryptographic protocols to exchange among peers recommendations about reliability of e-service providers. 
Trust values tend to change as the result of commercial interactions, since the outcome of each transaction may increase or decrease them. Hence it is reasonable to assume that the underlying trust graph is, in general, unknown or, even if it is known, trust degree, i.e. edge labels, are unknown.

The problem of searching a graph which is partially unknown has been studied in [11]. They consider the problem of computing shortest paths in a graph whose edges become known only when the search process arrives at edges endpoints. It is then proved that for general graphs the computational problem of devising a strategy producing a path with a given worst-case ratio to the optimum shortest path is P-SPACE complete ${ }^{5}$, while approximating the optimum worst-case ratio is a NP-hard problem. They also discuss a variant where the graph edges are known, but their labels become known only when reaching edges endpoints, and the same complexity results hold.

The concept of user point of view or context-aware computing is also adopted in the software engineering area, to describe agent behavior in ad-hoc mobile environments [6]. There, the concept of view is introduced as an abstraction of a particular agent's operating context, encompassing all the data that the agent can reach in the network. The view is defined by the agent as an egocentric (i.e., with reference to itself) function of the network topology and of properties of various entities in the network: in such a way an agent is able to adapt and tune its behavior according to the specific context where it is currently operating. Our work can be considered as a first step in devising algorithmic mechanisms supporting the implementation of context-aware middleware.

\section{Acknowledgments}

The authors would like to thank Enrico Nardelli and Maurizio Talamo for their useful discussions related to research issues here discussed.

\section{References}

[1] S. Braynov, and T. Sandholm. Contracting with uncertain level of trust. Computational Intelligence: special issue on Agent Technology for Electronic Commerce, 2002.

[2] G. Caronni. Walking the web of trust. In Proc. of 9th Workshop on Enabling Technologies (WETICE 2000), IEEE Computer Society Press, 2000.

[3] J. Coleman. Foundations of Social Theory. Harvard University Press, 1990.

${ }^{5}$ Remember that P-SPACE complexity class strictly includes NP 
[4] B.A. Hubermann, M. Franklin, and T. Hogg. Enhancing privacy and trust in electronic communities. In Proc. of the 1st ACM Conf. on Electronic Commerce (EC'99), 78-86, 1999.

[5] A. Josang, I.G. Pederson, and D. Povey. PKI Seeks a Trusting Relationship. In Proc. of ACISP 2000, Brisbane, Australia, 2000.

[6] C. Julien, and G.C. Roman. Egocentric Context-Aware Programming in Ad Hoc Mobile Environments. Technical Report WUCS-02-01, Washington University, Department of Computer Science, St. Louis (to appear in Proceedings of the 10th International Symposium on the Foundations of Software Engineering (FSE-10)).

[7] P. Ketchpel, and H. Garcia-Molina. Making Trust Explicit in Distributed Commerce Transactions. In Proc. 16th Int. Conf. Distributed Computing Systems, IEEE CS Press, Los Alamitos, California, 270-281, 1996.

[8] U. Maurer. Modelling a Public-Key Infrastructure. In Proc. of ESORICS 96 - 4th European Symposium on Research in Computer Security, Lecture Notes in Computer Science vol. 1146, pp. 325-350, Springer, 1996.

[9] S. Ortiz Jr. Will PKI Become Become a Key to Online Security ?. Computer, IEEE Computer Society, 33(12): 13-15, 2000.

[10] S. Paolini. Migliorare la Fiducia dell'Utente in Infrastrutture PKI: Un Approccio Architetturale. Tesi di Laurea, Università dell'Aquila, Dipartimento di Informatica, Italia, 2001.

[11] C.H. Papadimitriou, and M. Yannakakis. Shortest Paths Without a Map. TCS 84(1): 127-150, 1991.

[12] J. Su, and D. Manchala. Building Trust for Distributed Commerce Transactions. In Proc. 17th Int. Conf. Distributed Computing Systems, IEEE CS Press, Los Alamitos, California, 322-329, 1997.

[13] R. Yahalom, B. Klein, and Th. Beth. Trust Relationships in Secure Systems - A Distributes Authentication Prospective. In Proc. IEEE Conf. on Research on Security and Privacy, pp. 150-164, 1993. 